\title{
The Research on the Application of Computer Simulation in Competitive Aerobics Teaching and Training CHI Xiao-peng ${ }^{1}$
}

${ }^{1}$ Department of Physical Education \& Research, Beijing Foreign Studies University, Beijing 100089, China

\author{
Keywords:Computer simulation technology; Sports teaching; Aerobics; Application
}

\begin{abstract}
As the computer science develops rapidly, the computer simulation technology is becoming more and more mature. This technology is widely applied in different fields such as science, biology, architecture and education. In the perspective of the features and functions of computer simulation technology, this article uses competitive aerobics as an example, and researches into the application of this technology in physical education and exercise. Considering the characteristics of competitive aerobics, this article uses the empirical test as its methodology, and gives a detailed description of the application of this technology in the teaching and training of competitive aerobics. The result shows that this technology has brought revolutionary changes in the traditional and monotonous teaching and training method, and that coaches, students, and athletes all benefit from this technology; the use of this technology in this sport increases the training efficiency significantly and fully exploit the potential of this technology. This success can be used as a precedent for applications of this technology in other fields.
\end{abstract}

\section{Introduction}

Computer simulation is a comprehensive technical subject that is practice-oriented with strong applicability, and is widely applied in the field of education. It is first pervasively used in experimental classes like Physics, Chemic, Electronic Circuit, etc. Relevant simulation software gradually arises at the same time and has received great effects. But the application of computer simulation technology still holds little popularity in the domains of Physical Teaching, Athletic Training, etc.

So far, several problems still exist in applying computer simulation technology to Physical Training and Teaching field. Firstly, due to the diversity of Physical Technical Teaching and Training, no special simulation software for the Physical Training and Teaching field. Secondly, the complexity of simulation technology itself makes PE teachers find it difficult to employ simulation technology without technical supports from professionals. Furthermore, it's difficult to combine researchers with technical Sports Subjects. Thirdly, the differences between Sports Teaching and other teaching fields create a lack of experiences for references. Thus, introducing computer simulation technology into Sports training and teaching will produce a positive impetus to carry out the work of Sports Training and Teaching in the future, while giving full play to the advantages of computer simulation technology.

Therefore, bringing computer simulation technology into physical education will impact positively on the teaching and training of sports, and will expand the use of computer science into other areas. This paper starts with the characteristics of competitive aerobics, uses empirical test as its methodology and further examines the viability of its application in competitive aerobics and other fields.

\section{Purpose of Design}

2.1 Explore the viability of using computer simulation technology in physical education.

2.2 Use empirical test and examine the effect of using this technology in competitive aerobics, in order to inspire the application of this technology in other sports and diversify the methods of teaching and training. 


\section{Results and Discussion}

\section{Computer Simulation technology and its effects in teaching}

Computer Simulation technology is actually a technology that builds simulation models and conducts simulation experiments. It's a technique that combines environment (either realistic or imitative) to research, analyze and experiment by setting up the model of the research systems. As a research method and experimental technique, it is directly applied in system researches. We can use 3 basic activities to describe the relationship between the 3 key factors of Computer Simulation.

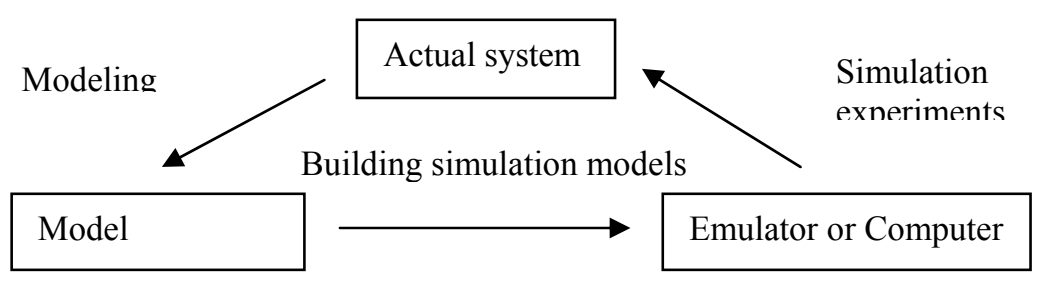

Figure 1 Relations among the Three Key Factors of Computer Simulation

Simulation technology possesses incomparable superiority of "authenticity" over other media in the field of Education. This "authenticity" is mainly provided with the following characteristics: Subject Immersion, Natural Interaction, Object Autonomy and Overall Perception. The most essential characteristic of simulation is users' immersion in virtual scenes. It needs to build a harmonious man-machine environment that closely resembles objective environment but is able to be immersed in and controlled while transcending the objective space-time. Those systems which can wholly or partially reach this aim, including all relevant techniques and methods of Natural Mimic and Lifelike Experience, are called simulation systems. According to the different forms of participation and different degrees of immersion of users, simulation systems can be divided into four categories: Simple Simulation System; Distributed Simulation System; Enhanced Simulation System; Immersion-Type Simulation System [2].

According to the pattern of human's memory, we need to experience a repeatedly process of learning to maintain what we've learned. But traditional class teaching fails to receive satisfying effects due to its disposable characteristics. Simulation training technology, however, is a environment that can give full play to students'creativeness. Students are able to learn independently without teachers and test their experiment schemes repeatedly until digest the knowledge thoroughly.

Simulation teaching (or simulation training) is a comprehensive multi-channel teaching method. When in simulation environments, students can fully mobilize their learning functions through channels of senses, sports and thoughts, and increase their learning effects dominantly. According to US Atlantic Simulation Company, teaching Psychologists have conducted comparison experiments between traditional teaching and simulation teaching. As is shown by the comparison between students' memorized contents after the two different methods, simulation lead to a memorization of $70 \%$ of the total content, compared to a $30 \%$ proportion after the students implement a traditional "teach and learn" method.

\section{Computer Simulation technology's application prospects in the field of Physical Teaching and Training.}

Physical teaching consists of theoretical teaching and practical teaching. The practicality of outdoor teaching activities broadens the prospects of Computer Simulation Technology in the field of Sports Teaching and Training. On one hand, we can arouse students' interest through simulated multimedia courseware produced to enrich teaching contents in theoretical teaching. On the other hand, in practical teaching, it may sometimes be difficult for teachers to demonstrate accurately due to the aging process and the deterioration of expertise techniques and physical condition. For students, they can only realize their performance from comments of teachers and peers. We can help students to recognize their shortcomings and to practice in virtual environment by inventing and designing simulation software. 


\section{The implementation of Computer simulation technology in Juvenile Aerobics Teaching and Training}

Competitive Aerobics gains universal popularity among juveniles because that it is a sport with music accompaniment. It pursues the combination of Power and beauty, music and action. Most schools have courses on Competitive Aerobics so far. Teachers, however, only concern on explanation and demonstration during the process of elaboration in the routine teaching and training. Furthermore, it may sometimes be difficult for teachers to demonstrate accurately due to the aging process and the deterioration of expertise techniques and physical condition. Normally students have to grasp the correct actions through repeated practice and continuous corrections from tutors. Although students can memorize the elaboration and demonstration given by their tutors on set, they can easily ignore the pace and correct position to be at while concentrating only on proficiency of the movement when they are practicing on their own. With the implement of simulation technologies, these problems can be easily solved. We can assist teaching process through the production of simulation software. In the process of teaching and training, lively and vivid demonstration rendered by simulation technology can help athletes master movements faster and more easily, so as to improve their technical skills. There are several dominant factors as listed below.

i) Aided Demonstration

Introducing computer simulation into practice courses of physical education can not only raise students' interest, but also provide a better solution to the interference to demonstrations in physical education caused by such factors as the short duration and anchorless feature (e.g. when having no contact with solid surface) of some movement, or the limitations of the tutor in both profession and accuracy affected by the aging process. The outcome of education could be improved by using a combined method of tutors giving demonstration on easy moves and computer-simulated footages elaborating difficult moves.

ii) Progress Manipulation

Tutors would embed sensors under the carpets on the training courts according to the key points in specific moves where the course of the athletes' movements is supposed to change dramatically (e.g. the beginning, the turning points or the end of a move). These sensors would be connected to a computer and set to the intended time to reach and time to linger. The feedback of the sensors would be reflected on the monitor in real time and can be played back after each move, showing mismatch of time or imperfection in students' moves, making it easier for students to amend or improve. For instance, when accomplishing Third Level Compulsory Routines in Competitive Aerobics, beginners usually move faster than they should and mismatch the pace of the music. In this case, tutors can connect multiple sensors placed in specific points in space to the computer. The computer would start logging as the student reaches one point, when at the same time; a red light connected to the computer would light up to give the student a stop signal. The student would then stop as suggested until a green light is on when the designed time to linger is reached, and the student would continue with her/his successive moves. Through a series of control given by the $\mathrm{red} /$ green lights, the overall pace and time in the progress can be manipulated.(As is shown in Figure 2)

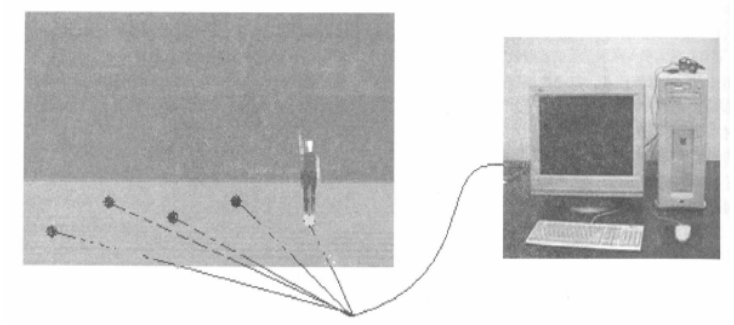

Figure 2 Computer Simulation Control of Aerobics Routines

iii) Simulated Practice

Computer simulation technology can be used to generate virtual character and environment. Tutors can provide the students with a lively virtual partner whose moves can be examined through 
intuitive and accurate 3D animations, which can help students to evaluate their own master of certain complex and constantly changing aerobics moves

\section{Conclusion and Suggestion}

Despite that computer simulation technology is still fresh, and that its application in physical education is still dawning, it has a bright future and considerable potential in the application to contemporary physical education and training due to the reasons that it can help construct variable educational patterns, that it better suits the multi-channel learning mechanism of humans, and that it can stimulate learning efficiency. Although computer simulation technology and its system hardware are still expensive in the current situation, we believe, provided thatcomputer simulation technology is developing rapidly while the hardware price is dropping, that as a new teaching platform, computer simulation technology, with its huge advantages and potentials in education and research, will attract attention of and gain popularity in the stage of physical education and training.

Genetic algorithm is widely used in the multi-objective optimization problems with global search capability and robustness[7]. In order to solve the problem (3), we encode the upper variable values using real coding scheme, and give a fitness function based on non-dominated solutions sorting method and crowding distance, it can distinguish different individuals effectively. Our algorithm is developed:

\section{References}

[1] Wang Zhengzhong, TuRenshou (1991): National Defense Industry Press. Contemporary modern computer simulation technology and Application [M]. Beijing, China.

[2] Luo Xiaodong, Wang Qinlong: The Application of Virtual Reality Technology in Teaching [J], $8: 53-54,2003$.

[3] HeKekang (January, 2005). Theory and Method of the Deep integration of information technology with Curriculums [J].E-education Research, 1

[4] WuChongguang Simulation and Education, Journal of System Simulation [J], 6: 1285$1288,2005$.

[5] DaiYingming. Study on the Application of Virtual Reality Technology in Teaching [J], Information technology, 8: 86-88,2006.

[6] PeiYanyang (July 2007). The Application of Virtual Laboratory Based on Web in College Experimental Teaching [J]. Popular Science \& technology.

[7] Hu Ming (January 2001). Research on the Application of Computer Simulation Technology in Physics Teaching Process[J]. Computer Engineering Science.

[8] RenJianhua, Ran Ming (September 2006). Brief Analysis on Full Interactive Intelligent Chemistry Courseware and Its Realizing Method [J].Chemistry Teaching.

[9]Guo Shaoqing (2002). The Integration of Information Technology Education and Curriculum [M].Chinese Personnel Press. Beijing, China.

[10] Liu Yuan, Chen Yan jun, Ran Ming . Brief Probe into Development of Chemical Simulation Teaching Courseware [J]. China Education IFO, 32-34,2007.

[11] LuoXiangnan, Hu Pingya (2006). Interactive Function Design Based on Flash Simulation Experiment Courseware [J]. Chinese Science and Technology Information, 13. 